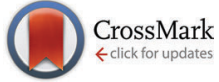

Cite this: Chem. Commun., 2015, 51, 14485

Received 22nd June 2015, Accepted 3rd August 2015

DOI: $10.1039 / \mathrm{c} 5 \mathrm{cc} 05104 \mathrm{e}$

www.rsc.org/chemcomm

\section{A novel detection technique of polyamide binding sites by photo-induced electron transfer in ${ }^{\mathrm{Br} U}$ substituted DNA $\dagger$}

\author{
Abhijit Saha, ${ }^{a}$ Fumitaka Hashiya, ${ }^{a}$ Seiichiro Kizaki, ${ }^{a}$ Sefan Asamitsu, ${ }^{a}$ Kaori Hashiya, ${ }^{a}$ \\ Toshikazu Bando a and Hiroshi Sugiyama*ab
}

\begin{abstract}
We report the photochemistry of ${ }^{\mathrm{Br}} \mathrm{U}$ substituted DNA as a versatile platform to investigate the binding sites of pyrene conjugated pyrrole imidazole polyamides (PIPs). The results suggest that the approach can be used on a routine basis for the screening of polyamide binding sites.
\end{abstract}

Replacement of thymine by isosteric 5-bromouracil $\left({ }^{\mathrm{Br}} \mathrm{U}\right)$ in DNA is attractive since it does not affect the functionality of the resulting DNA due to the similar sizes of $\mathrm{Br}(1.95 \AA)$ and methyl (2 $\AA^{1} .{ }^{1}$ As a result of this modification, it greatly increases the photosensitivity with respect to protein-nucleic acid crosslinks, ${ }^{1,2}$ single- and double-strand breaks, and the creation of alkali-labile sites. ${ }^{3-7}$ The chemistry of strand cleavage after irradiation at $302 \mathrm{~nm}$ of ${ }^{\mathrm{Br}} \mathrm{U}$-labeled DNA has been investigated based on the photodegraded products of a model hexamer $\mathrm{d}\left(\mathrm{GCA}^{\mathrm{Br}} \mathrm{UGC}\right)_{2} \cdot{ }^{8}$ The abstraction of hydrogen by the uracil-5-yl radical $\left(\mathrm{U}^{\bullet}\right)$, a powerful hydrogen abstractor, generated from the ${ }^{\mathrm{Br}} \mathrm{U}$ anion radical by eliminating the bromide ion, is the main photochemical event in such photoinduced strand cleavage. The isolation of two kinds of photodegraded products has pointed out two possible ways of hydrogen abstraction from the deoxyribose moiety at the $5^{\prime}$ position. ${ }^{9}$ The abstraction of hydrogen from the $\mathrm{C}^{\prime}$ position generates 2-deoxyribonolactone with the release of $\mathrm{A}$, and abstraction from the $\mathrm{C}^{\prime} \alpha$ position generates an erythrosecontaining site. ${ }^{9}$ The detailed mechanism of photodamage in ${ }^{\mathrm{Br}} \mathrm{U}$-labeled DNA has recently been clarified further with the identification of the hot-spot sequences $5^{\prime}-\mathrm{G} / \mathrm{C}[\mathrm{A}]_{n=1,2,3}{ }^{\mathrm{Br}} \mathrm{U}^{\mathrm{Br}} \mathrm{U}-3^{\prime}$ and their reverse $3^{\prime} \rightarrow 5^{\prime}$ sequence. ${ }^{10,12}$ Initially, the A residue adjacent to ${ }^{\mathrm{Br}} \mathrm{U}$ was considered to be the electron donor; however, subsequent experiments confirmed that the $\mathrm{G}$ residue, which has the lowest oxidation potential among the four bases, is the electron donor. ${ }^{8,11}$ Moreover, the A bridge helps to prevent

\footnotetext{
${ }^{a}$ Department of Science, Graduate School of Science, Kyoto University, Sakyo, Kyoto 606-8501, Japan.E-mail: hs@kuchem.kyoto-u.ac.jp

${ }^{b}$ Institute for Integrated Cell-Material Sciences (iCeMS), Kyoto University,

Yoshida Ushinomiya-cho, Sakyo, Kyoto 606-8502, Japan

$\dagger$ Electronic supplementary information (ESI) available. See DOI: 10.1039/c5cc05104e
}

rapid back electron transfer. ${ }^{11}$ In contrast to our observations, direct strand cleavage has also been reported after aerobic and anaerobic photolysis. ${ }^{13,14}$ The proposed mechanism for direct strand breakage also involves hydrogen abstraction by $\mathrm{U}^{\bullet}$ from the $\mathrm{C}^{\prime}$ and $\mathrm{C} 2^{\prime}$ positions at $5^{\prime}$, albeit via different mechanistic pathways. Recently, successful quenching of highly reactive $\mathrm{U}^{\bullet}$ in the hot-spot sequences was shown by supplementation with an excess amount of the hydrogen donor tetrahydrofuran (THF), to stop intrastrand hydrogen abstraction. ${ }^{12}$ The elucidation of the mechanistic details of the photochemistry of ${ }^{\mathrm{Br}} \mathrm{U}$-labeled DNA has attracted much attention due to its biological and technological applications.

Pyrrole imidazole polyamides (PIPs) are synthetic small molecules that can bind to the minor groove in a sequence specific manner. These molecules gained much interest in biology since they have been shown to regulate gene expression. ${ }^{15}$ Footprinting and affinity cleavage techniques have guided the development of the pairing rule of PIPs and it is likely to be the side-by-side stacked ring pairing such as an Im opposite to $\mathrm{Py}(\mathrm{Im} / \mathrm{Py}$ ) can recognize a G/C base pair from the $\mathrm{C} /$ $\mathrm{G}$ base pair, whereas a Py located opposite to Py (Py/Py) recognizes either an $\mathrm{A} / \mathrm{T}$ or $\mathrm{T} / \mathrm{A}$ base pair. ${ }^{15-21}$ It is common to see a polyamide binding preferentially to a high-affinity binding site in the presence of several potential binding sites, although the underlying mechanism remains unclear. Therefore, it is crucial to screen a library of potential binding sites to identify real highest-affinity binding sites. The application of PIPs in biology will require knowledge of its sequence specificity and has necessitated a more advanced screening technique that can be routinely used in higher throughput. Although affinity cleavage methods explored polyamide-binding affinity to a great extent, they are partially sensitive and the synthesis challenges regarding the attachment of EDTA to polyamide render them challenging methods for the determination of binding sites. Because of easier pyrene conjugation and ${ }^{\mathrm{Br}} \mathrm{U}$-substituted DNA being more sensitive than native DNA, the combined output of the current approach is more facile and sensitive than previous approach. 


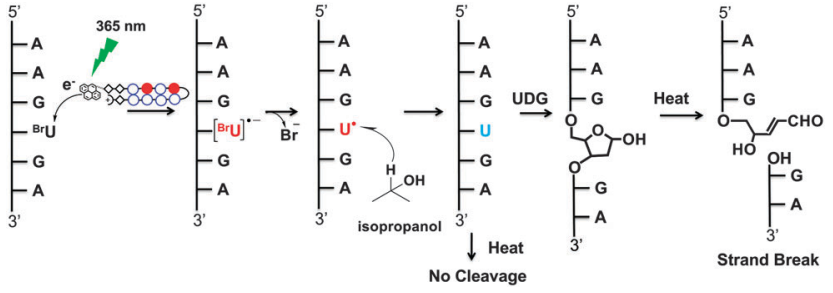

Fig. 1 Generation of the highly reactive uracil-5-yl radical (shown in red) by ${ }^{\mathrm{Br}} \mathrm{U}$, an efficient electron acceptor, by capturing an electron. In the presence of an excess of the hydrogen atom donor isopropanol, the radical species can be quenched to uracil (shown in blue). Treatment with the uracil DNA-glycosylase (UDG) enzyme can convert uracil to the heatlabile Ap site selectively, which, under heating conditions (at $95^{\circ} \mathrm{C}$ ), causes strand breakage.

To test this hypothesis, we prepared two long, fully ${ }^{\mathrm{Br}} \mathrm{U}$-substituted DNA fragments (381 bp: DNA1 and $298 \mathrm{bp}$ : DNA2) using PCR and designed four pyrene conjugated polyamides (1-4, Fig. 2) to check their binding affinity and specificity by electron injection. In early studies, it has been well characterized that pyrene serves as an electron donor in DNA mediated excess electron transfer. ${ }^{2-26}$ Thus, after irradiation at $365 \mathrm{~nm}$ for a minimum of $5 \mathrm{~s}$, each pyrene PIP injected an electron into ${ }^{\mathrm{Br}} \mathrm{U}$ residues at their binding sequences. Using high-resolution gel electrophoresis, the site of electron injection was identified from the strand break caused by the sequenceselective generation of the $\mathrm{U}^{\bullet}$ radical. A photoreaction scheme of the photo-induced electron injection in ${ }^{\mathrm{Br}} \mathrm{U}$-substituted DNA is shown in Fig. 1.

From the analysis of DNA1 (top and bottom strands separately) it was revealed that polyamide $\mathbf{1}$ (pyrene- $\beta \beta$ PyImPyIm- $\gamma$ PyPyPyPy $\beta D p)$, which can recognize $5^{\prime}$-WCWCWW-3' and $5^{\prime}$-WWGWGW-3' ( $\left.\mathrm{W}=\mathrm{A} / \mathrm{T}\right)$ sequences, gives strand cleavages in three match sequences (sites 1,3 , and 5) via electron injection (shown in red in Fig. 3 and 4 and the ES1 $\dagger$ ). Moreover, two 1 bp mismatch sites (sites 2 and 4 ) were detected by strand cleavage. The amount of strand cleavage at the mismatch sites was almost identical to that detected at the match sites. In DNA2 (Fig. S2, ESI $\dagger$ ) 1 exhibited strand cleavages at four match sequences (sites 1-4). In addition, there were two strong strand cleavage events at sites 5 and 6 , which were considered as 1 bp mismatch sites. Strand cleavage at the mismatch sites of $\mathbf{1}$ was consistently strong, as revealed by both DNA fragments, which clearly indicates that $\mathbf{1}$ has low sequence specificity. Moreover, it has been noticed that the amount of strand cleavage was not identical at all match sites. This might result from sequence preference among many potential binding sites, although the exact reason is unknown.

Polyamide 2 (pyrene- $\beta \beta$ PyPyPyIm- $\gamma$-PyImPyPy $\beta$ Dp) can recognize $5^{\prime}$-WCGWWW-3' and 5'-WWWCGW-3' sequences. However, in the DNA1 fragment, it did not have any match sequences. However, the photoreaction revealed the presence of several strong strand cleavage events at sites $6,7,9,10$, and 11 , which are considered as 1 bp mismatch sites, Fig. 3 and 4 and ES1. $\dagger$ Interestingly, a medium-strength strand cleavage at site 8 shifted our attention toward the reverse orientation, as it is a $1 \mathrm{bp}$ mismatch reverse
A
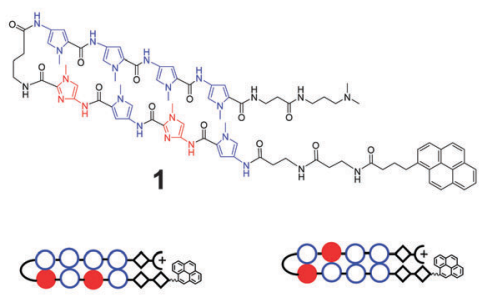

1

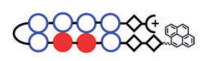

3

$O: N$-methylpyrrole $: N$-methylimidazo

$C$ : $\gamma$-amino butyric acid $\diamond$ : $\beta$-alanine
B

Forward Orientation $\mathrm{N} \longrightarrow \mathrm{C}$

5'-W C G W W W-3'

$3^{\prime}-W$ G C W W W-5'

$2^{W} \quad W=A / T$

C

Reverse Orientation

C-N terminus, $5^{\prime}-3^{\prime}$

$\mathrm{C} \longrightarrow \mathrm{N}$

5'-พ G C พ พ พ-3' observed
N-C terminus, $5^{\prime}-3^{\prime}$
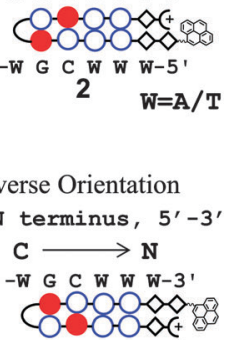

3'-W C G W W W-5'

Fig. 2 (A) Chemical structures of pyrene polyamides 1 , and similarly cartoon of 1-4. (B) Forward binding orientation in which the $N$ to $C$ terminus of polyamides aligns with $5^{\prime} \rightarrow 3^{\prime}$ DNA. (C) Reverse binding orientation in which the $\mathrm{C}$ to $\mathrm{N}$ terminus of polyamides aligns with $5^{\prime} \rightarrow 3^{\prime}$ DNA.

binding site of 2 (shown in green, Fig. 4). Generally, polyamides preferentially align with the $\mathrm{N}$ terminus of the antiparallel DNA strand, with its $5^{\prime}$ end in the $\mathrm{N} \rightarrow \mathrm{C}, 5^{\prime} \rightarrow 3^{\prime}$ orientation, which is known as the forward orientation. In some cases, polyamides can also recognize the reverse orientation, for example $\mathrm{C} \rightarrow \mathrm{N}$, $5^{\prime} \rightarrow 3^{\prime}$, as shown in Fig. 2(B) and (C). ${ }^{27}$ Strand cleavage in the DNA2 fragment, Fig. S2A (ESI $\dagger$ ), was more interesting, as the reverse orientation binding was consistently observed at site 10 , which was a full match reverse binding site of 2, Fig. S2(C) (ESI $\dagger)$. The amount of strand cleavage at site 10 was almost identical to that observed at match sites. This indicates that 2 can bind in the reverse orientation efficiently. As 2 has match sequences in DNA2, photoirradiation also gave cleavage at those sites, such as sites 7 and 9. In addition, strong strand cleavage at $1 \mathrm{bp}$ mismatch sites was also observed at sites 8 and 11. It should be noted that after incubating DNA1-2 fragments with 2 subsequent photoirradiation results in consumption of almost nearly $90 \%$ of the total amount of unreacted DNA under conditions that were similar to that used for other polyamides (Fig. 3 and Fig. S2(A), ESI $\dagger$ ). This indicates its strong nonspecific binding preferences.

Next, we designed polyamide 3 (pyrene- $\beta \beta$ PyImImPy- $\gamma$ PyPyPyPy $\beta D$ p) to target $5^{\prime}-\mathrm{WWGGWW}-3^{\prime}$ and $5^{\prime}-\mathrm{WWCCWW}-3^{\prime}$ sequences. The location of these sequences in DNA1 was completely different from the cleavage sites of $\mathbf{1}$ and 2 . Thus, strand cleavage at the target site of $\mathbf{3}$ was deemed as crucial to support electron injection based on sequence specificity. Interestingly, strong strand cleavage at the target sites of $\mathbf{3}$, such as sites 15 and 16, Fig. 3, was detected, which strongly supports the sequence selectivity. As polyamides targeting $6 \mathrm{bp}$ DNA sequences can also bind to mismatch sequences, cleavage at $1 \mathrm{bp}$ mismatch sites, such as sites $12-14$, by 3 was normal. However, the amount of cleavage at these sites was lower than that detected at match sites, which also indicates that 3 can differentiate between match and mismatch sites under similar conditions. In contrast, in DNA2 there was no match sequence; 


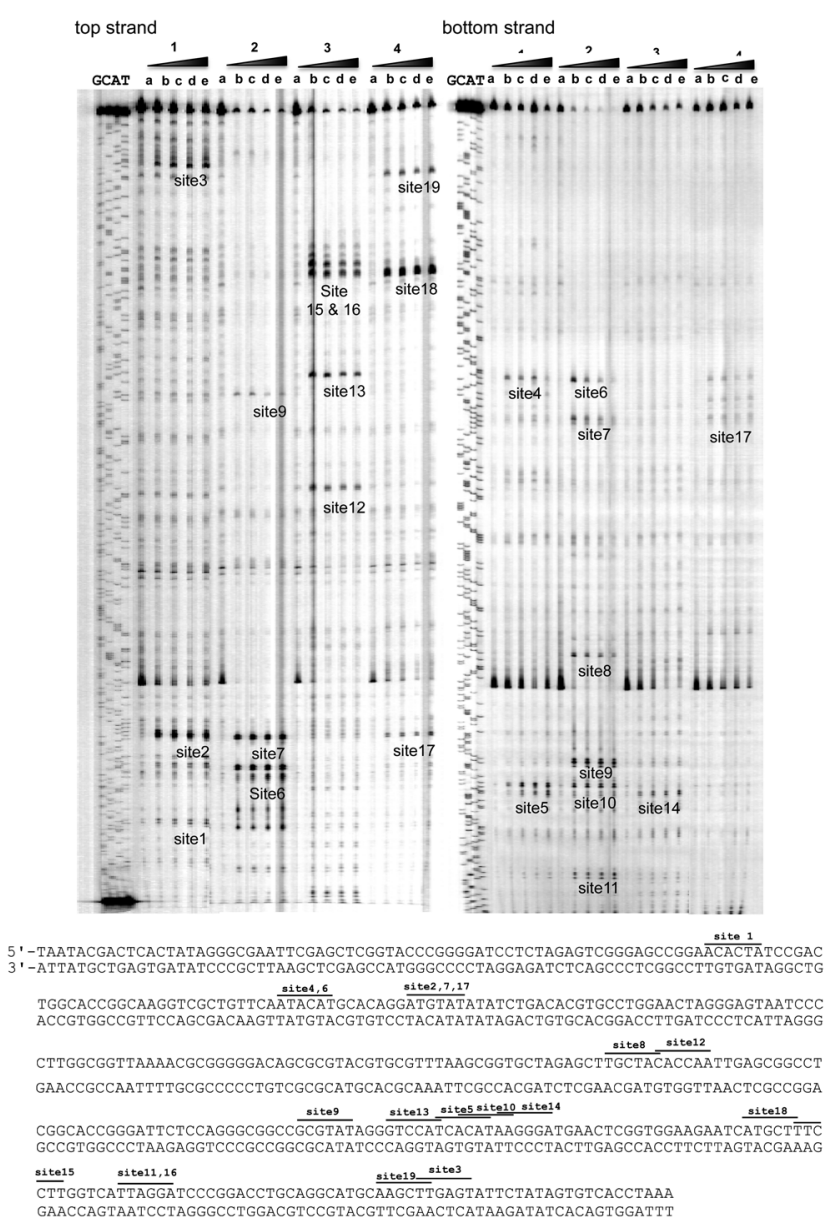

Fig. 3 Slab gel sequencing analysis of a $6 \%$ denaturing (7 M urea) polyacrylamide gel for the DNA1 (381 bp) after photoreaction using polyamides 1-4 Lanes a-e, photoirradiation periods of $0,5,10,15$, and $30 \mathrm{~s}$, respectively, at $365 \mathrm{~nm}$ UV using $10 \mathrm{~nm}$ DNA and $100 \mathrm{~nm}$ polyamide. Top- and bottomstrand analyses are shown. The sequence of DNA1 is shown in which all Ts are replaced with ${ }^{\mathrm{Br}} \mathrm{U}$.

however, three extremely weak strand cleavage events, which were considered as $1 \mathrm{bp}$ mismatch sequences (sites 12-14, Fig. S2, ESI $\dagger$ ), were observed. This observation was consistent in both DNA fragments.

In a similar way, we designed polyamide 4 (pyrene- $\beta \beta$ PyImPyPy- $\gamma$-PyImPyPy $\beta D p$ ), to target $5^{\prime}$-WWGCWW-3' sequences because of their unique places in both DNA fragments. Strand cleavage events at sites 18 and 19 confirmed its match sequences in the DNA1 fragment, Fig. 3 and 4 and ES1. $\dagger$ Moreover, a weak strand cleavage observed at site 17 confirmed its $1 \mathrm{bp}$ mismatch sequence. In DNA2 (Fig. S2, ESI $\dagger$ ), site 15 was the only match sequence for $\mathbf{4}$ in the entire DNA fragment, and strong strand cleavage at that sequence further confirmed the sequence selectivity. In addition, two $1 \mathrm{bp}$ mismatch sequences, sites 16 and 17, were also cleaved after the photo-irradiation. It should be noted that, although these types of polyamides can easily bind to $1 \mathrm{bp}$ mismatch sequences, the number of mismatches and the amount of cleavage in the case of $\mathbf{4}$ were lower. This implies its higher sequence specificity among the four designed polyamides. Again it is to be noted that whether pyrene has any influence on polyamide binding
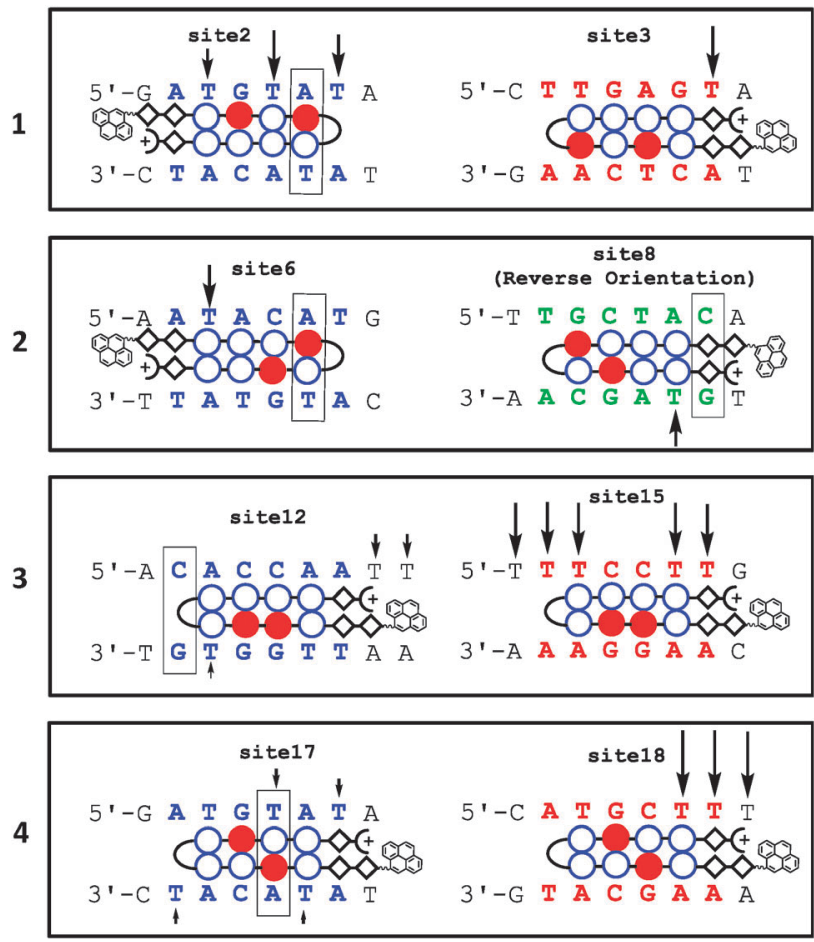

Fig. 4 Representative examples of electron injection in DNA1 from sites 1-5, 6-11, 12-16, and 17-19 by polyamides $\mathbf{1}, \mathbf{2}, \mathbf{3}$ and $\mathbf{4}$, respectively, are shown. The remaining examples are shown in Fig. S1 (ESI†). Binding sites are shown in different colors: red indicates a match site, blue indicates a $1 \mathrm{bp}$ mismatch site, and green indicates a reverse binding site; the boxes at mismatch sites indicate the mismatched base pair. Arrows indicate the strand-break site, with intensity.

specificity or not is unknown. However, pyrene is known to bind DNA relatively non-specifically by intercalating between the base pairs,$^{28}$ thus the absence of such cleavages suggests that pyrene conjugation with polyamide might not appreciably influence the specificity. Because in the present study we admitted that binding affinity, binding orientation, and specificity can be estimated by strand cleavage via electron injection, we performed SPR on biotinylated $\mathrm{DNA}^{29}$ containing those particular sequences of match, mismatch, and reverse binding sites, for further validation of the results.

First, we examined the binding affinity of polyamide 1 at match and $1 \mathrm{bp}$ mismatch sites. The results are shown in Table 1 . The $K_{\mathrm{D}}$ values for match and mismatch sites were $5.96 \times 10^{-8}$ and $1.05 \times$ $10^{-7}(\mathrm{M})$, respectively. The specificity between these two values was just 1.76. These values indicate almost a similar binding affinity in match and mismatch sites that were observed from strand cleavage in both DNA fragments. As photoirradiation of DNA incubated with 2 pointed out the possibility of binding in the reverse orientation, binding affinity in forward and reverse orientations was further examined by SPR. The $K_{\mathrm{D}}$ value in the forward and reverse orientations of 2 was $5.13 \times 10^{-8}$ and $1.51 \times 10^{-7}(\mathrm{M})$, respectively, and the specificity was just 2.94 . This result suggests that 2 can bind in the reverse orientation at site 10 in DNA2 with almost the same affinity as that detected in the forward orientation; see Fig. S2A (ESI $\dagger$ ). The $\sim 3.5$ times higher $k_{\mathrm{a}}$ value observed 
Table 1 SPR analysis validating the binding affinity

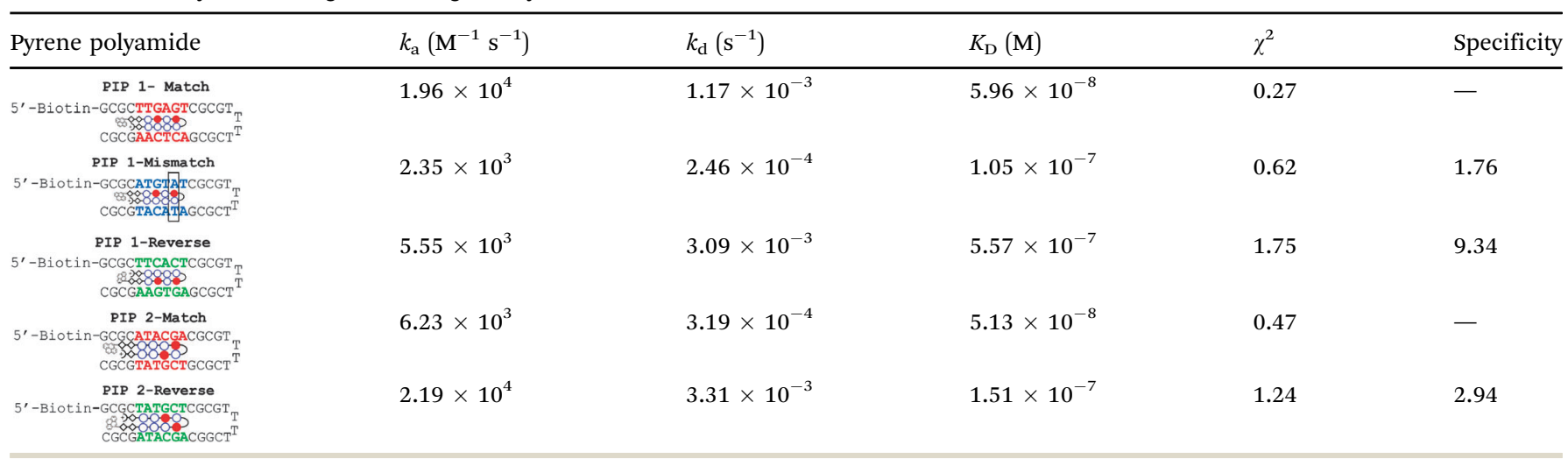

in the reverse site compared with the forward site also indicates that 2 can access the reverse sequence more efficiently. We also checked the reverse binding affinity of $\mathbf{1}$, as it contains several reverse binding sites in both DNA fragments. However, those reverse sequences overlapped with forward sequences; for example, site 1 in Fig. 3. Polyamide 1 exhibited a $K_{\mathrm{D}}$ value of $5.57 \times 10^{-7}(\mathrm{M})$ in the reverse orientation, and its specificity with respect to the forward orientation was 9.34. This value indicates a significant low binding affinity for $\mathbf{1}$ in the reverse orientation. Moreover, the $k_{\mathrm{a}}$ value for the forward orientation was $\sim 3.5$ times higher than that for the reverse orientation, which is completely opposite to what was observed for 2 .

In conclusion, we have demonstrated that the photochemistry of ${ }^{\mathrm{Br}} \mathrm{U}$-substituted DNA can be used to detect the binding site of PIPs. Using this technique, we have successfully analyzed the binding sites of four pyrene conjugated PIPs in two long ${ }^{\mathrm{Br}} \mathrm{U}$-substituted DNA fragments (381 bp and 298 bp) by photo-induced electron injection and estimated their binding affinity, specificity and orientation preferences. Using high-resolution denaturing gel electrophoresis, the site of electron injection was analyzed. PAGE analysis revealed a unique pattern of electron injection from polyamides 1-4 in both DNA fragments. Our results suggest that $\mathbf{1}$ and $\mathbf{2}$ inject electrons with low sequence specificity and $\mathbf{3}$ and $\mathbf{4}$ inject electrons with high sequence specificity. Moreover, using this technique we can also detect reverse orientation binding sites, as observed for 2 . As it is crucial to scan libraries of many potential binding sites to identify binding sites with the highest affinity, this platform would be a useful screening tool in the design and development of PIPs.

\section{References}

1 M. C. Willis, B. J. Hicke, O. C. Uhienbeck, T. R. Cech and T. H. Koch, Science, 1993, 262, 1255-1257.

2 B. J. Hicke, M. C. Willis, T. H. Koch and T. R. Cech, Biochemistry, 1994, 33, 3364-3373.

3 R. Ogata and W. Gilbert, Proc. Natl. Acad. Sci. U. S. A., 1977, 74, 4973.
4 F. Krasin and F. Hutchinson, Biophys. J., 1978, 24, 657-664.

5 K. Suzuki, M. Yamauchi, Y. Oka, M. Suzuki and S. Yamashita, Nat. Protoc., 2011, 6, 134-139.

6 F. Krasin and F. Hutchinson, Biophys. J., 1978, 24, 645-656.

7 H. Sugiyama, Y. Tsutsumi, K. Fujimoto and I. Saito, J. Am. Chem. Soc., 1993, 115, 4443-4448.

8 H. Sugiyama, Y. Tsutsumi and I. Saito, J. Am. Chem. Soc., 1990, 112, 6720-6721.

9 H. Sugiyama, K. Fujimoto and I. Saito, Tetrahedron Lett., 1996, 37, 1805-1808.

10 T. Watanabe, T. Bando, Y. Xu, R. Tashiro and H. Sugiyama, J. Am. Chem. Soc., 2005, 127, 44-45.

11 T. Watanabe, R. Tashiro and H. Sugiyama, J. Am. Chem. Soc., 2007, 129, 8163-8168.

12 F. Hashiya, A. Saha, S. Kizaki, Y. Li and H. Sugiyama, Nucleic Acids Res., 2014, 42, 13469-13473.

13 G. P. Cook and M. M. Greenberg, J. Am. Chem. Soc., 1996, 118, 10025-10030.

14 G. P. Cook, T. Chen, A. T. Koppisch and M. M. Greenberg, Chem. Biol., 1999, 6, 451-459.

15 P. B. Dervan, Bioorg. Med. Chem., 2001, 9, 2215-2235.

16 M. W. Van Dyke and P. B. Dervan, Science, 1984, 225, 1122.

17 P. B. Dervan, Science, 1986, 232, 464.

18 M. Brenowitz, D. E. Senear, M. A. Shea and G. K. Ackers, Methods Enzymol., 1986, 130, 132.

19 M. Brenowitz, D. E. Senear, M. A. Shea and G. K. Ackers, Proc. Natl. Acad. Sci. U. S. A., 1986, 83, 8462.

20 D. F. Senear, M. Brenowitz, M. A. Shea and G. K. Ackers, Biochemistry, 1986, 25, 7344.

21 J. W. Trauger and P. B. Dervan, Methods Enzymol., 2001, 340, 450-466.

22 P. Kaden, E. Mayer-Enthart, A. Trifonov, T. Fiebig and H.-A. Wagenknecht, Angew. Chem., Int. Ed., 2005, 44, 1636.

23 H.-A. Wagenknecht, Angew. Chem., Int. Ed., 2003, 42, 2454.

24 R. Tashiro, A. Ohtsuki and H. Sugiyama, J. Am. Chem. Soc., 2010, 132, 14361-14363.

25 T. L. Netzel, M. Zhao, K. Nafisi, J. Headrick, M. S. Sigman and B. E. Eaton, J. Am. Chem. Soc., 1995, 117, 9119-9128.

26 H. Morinaga, T. Takenaka, F. Hashiya, S. Kizaki, K. Hashiya, T. Bando and H. Sugiyama, Nucleic Acids Res., 2013, 41, 4724-4728.

27 J. L. Meier, A. S. Yu, I. Korf, D. J. Segal and P. B. Dervan, J. Am. Chem. Soc., 2012, 134, 17814-17822.

28 H.-C. Becker and B. Nordén, J. Am. Chem. Soc., 1999, 121, 11947-11952.

29 BIA Evaluation Software Handbook, Version 3, Biacore AB, Rapsgatan, Sweden, 1999. 